\title{
Isospora carliae sp. n. (Apicomplexa: Eimeriidae) from the skink Carlia rhomboidalis (Peters) from Daintree Forest, North Queensland, Australia: description and fine-structural account of endogenous development
}

\author{
Ilan Paperna \\ Department of Animal Sciences, Faculty of Agricultural, Food and Environmental Quality Sciences, The Hebrew University of \\ Jerusalem, Rehovot 76-100, Israel
}
Key words: Isospora carliae, Carlia rhomboidalis, Australia, oocysts, ultrastructure, intranuclear endogenous stages

\begin{abstract}
Isospora carliae sp. n. is described from the blue-throated rainbow skink Carlia rhomboidalis (Peters), from Daintree Forest, North Queensland, Australia. Oocysts are ellipsoidal, 16.8-21.0 $\times 12.6-15.4 \mu \mathrm{m}$ in size, with their two sporocysts, 9.0$14.0 \times 7.0-9.24 \mu \mathrm{m}$ in size, positioned along the wide axis. Sporozoites contain a distinct refractile body and are accompanied by a residuum. All endogenous development occurs within the host-cell nucleus. Nuclei are sometimes invaded by several merozoites, but only infections by a single parasite persist. Nuclei lodging meronts, mature microgamonts and premature macrogamonts have an elongate shape. Some meronts exhibit a membrane-bound cytoplasmic inclusion that contains many micronemes.
\end{abstract}

Cannon (1967) described two species of Isospora Schneider 1881 from Australian skinks. Two additional species were described by Finkelman and Paperna (1994). Some species of Isospora undergo endogenous development in the cytoplasm of their epithelial host cell, while others, such as I. cryptoblephari Finkelman et Paperna, 1994, develop inside the nucleus of their host cell. Although intranuclear and intracytoplasmic modes of development could constitute a distinctive taxonomic characteristic, most published descriptions of Isospora species are restricted to oocyst-sporocyst data. Intranuclear species of Isospora have been also described from skinks in the USA (Atkinson and Ayala 1987), the Canary Islands (Matuschka 1989) and Egypt (El Toukhy et al. 1996).

Several fine-structural images from the presently described Isospora species have been presented in an overview communication which illustrates the finestructural divergence among intranuclear species of Isospora from reptilian hosts (Paperna and Finkelman 1998, figs. 9, 22, 35, 36, 43, 44). In this communication, we present a formal description of Isospora carliae $\mathrm{sp}$. n. from the rainforest blue-throated rainbow skink Carlia rhomboidalis (Peters), with further details on its fine-structural organisation.

\section{MATERIALS AND METHODS}

Two specimens of $C$. rhomboidalis were collected in Daintree rain forest in North Queensland, Australia, in August
1986, and six in August 1988. The skinks were left to defecate inside a one-litre glass container. For microscopic examination, faeces were macerated in tap water. Faeces of two skinks from the 1986 collection and two from 1988 contained Isospora sp. oocysts [as well as species of Eimeria (s.l.) and of Choleoeimeria from the gall bladder, data to be reported elsewhere]. The infected faeces were preserved in $10 \%$ buffered neutral formalin (BNF). Infected skinks were euthanized, and their tissues were fixed for histology; the gut of one of the infected skinks from 1988 was fixed for electron microscopy. Uninfected skinks were released to their habitat.

For light microscopy (LM), portions of the gut were fixed in BNF and embedded in glycol methacrylate (GMA) medium (Agar Scientific, Stansted, UK). Sections of GMA-embedded material were cut to $2.0-3.0 \mu \mathrm{m}$ with a glass knife on a JB4 microtome. Some were stained with Meyer's haemalum and eosin; others were post-fixed in aqueous Bouin's solution for $20 \mathrm{~min}$, washed in $70 \%$ ethanol until colourless and stained in Giemsa (10\%, in $\mathrm{pH} 7.4$ phosphate buffer).

For transmission electron microscopy (TEM), small pieces of intestine were fixed in $2.5 \%$ glutaraldehyde in cacodylate buffer $(0.1 \mathrm{M}, \mathrm{pH} 7.4)$ for $24 \mathrm{~h}$ at $4^{\circ} \mathrm{C}$, rinsed repeatedly in the same buffer, post-fixed in $1.0 \%$ osmium tetroxide in the same buffer for $1 \mathrm{~h}$, rinsed in the same buffer, dehydrated in graded alcohols and embedded in Agar 100® resin (Agar Scientific Ltd.). Thin sections, cut on a Reichert Ultracut ultratome with a diamond knife, were stained on grids with uranyl acetate and lead citrate and examined in a JEOL 100CX TEM. Semithin sections prepared from Agar 100® resin-embedded tissue were stained with toluidine blue. All measurements are given in $\mu \mathrm{m}$.

Address for correspondence: I. Paperna, Department of Animal Sciences, Faculty of Agricultural, Food and Environmental Quality Sciences, The Hebrew University of Jerusalem, Rehovot 76-100, Israel. Phone: ++972 89489 945; Fax: ++972 89465 763; E-mail: ipaperna@agri.huji.ac.il 


\section{RESULTS}

\section{Isospora carliae sp. n.}

\section{Description}

Exogenous stages $(\mathrm{n}=13$, measured live, Fig. 1). Oocysts ellipsoidal, with two sporocysts positioned along the wide axis ("lateral position"), 16.8-21.0 $\times$ 12.6-15.4 (18.0 $\pm 1.4 \times 14.1 \pm 0.7)$ in size. Oocyst wall smooth and colourless, lacking micropyle and polar granule. Sporulated sporocysts 9.0-14.0 × 7.0-9.2 (11.5 $\pm 1.9 \times 7.8 \pm 0.9$ ), oval, with colourless wall. Stieda and substieda bodies at one apex accompanied by a residuum. Each sporozoites with a conspicuous refractile body.

Endogenous stages viewed by LM (not illustrated). All endogenous stages occurred within nuclei of the host's gut-epithelium in the posterior part of the small intestine. The parasitized nucleus was consumed gradually; its nucleolus persisted to the late stage of parasite development. Meront stages were not seen. Premature microgamonts, with nine detectable nuclei, were 16.8 $18.2 \times 9.8-9.8(\mathrm{n}=5)$. Some mature microgamonts, $18.2 \times 9.8$ in size $(\mathrm{n}=5)$, developed only a few (nine per section) microgametes; others, of the same or smaller size $(9.8-11.2 \times 5.6)$, yielded larger numbers of microgametes (more than 25 per section). Premature macrogamonts were 7.0-8.4 $\times 4.2-7.0$ in size $(\mathrm{n}=5)$. Zygotes (or young oocysts) containing many amylopectin granules and wall-forming bodies ranged from $9.8 \times 8.4$ to $13.0 \times 8.4$ in size $(\mathrm{n}=5)$.

\section{Fine structure of endogenous stages}

Some nuclei contained several merozoites (four are seen in Fig. 2), but usually accommodated only a single meront at later stages. Fig. 3 illustrates a young intranuclear uninucleate meront. The young meront's cytoplasm contained a single lipid vacuole, several mitochondria, a Golgi structure, a few micronemes and electron-dense vesicles (Fig. 3). Some multinucleate premature meronts disclosed several junction sites between the parasitophorous vacuole and the nucleolemma, in some, the nucleolemma at the junction site was accompanied by a small electron-dense structure of unknown nature (Fig. 4). In addition to the lipid vacuole, the cytoplasm of these more differentiated meronts contained smaller vacuoles (forming storage bodies?) and electron-dense vesicles (Fig. 4). A zoite-like structure, seen as an inclusion within the meront's cytoplasm, was bound by a bilayered membrane and contained numerous micronemes (Figs. 4, 5), with either a dense granular substance which contained a mitochondrion and enclosed an electron-dense core (Fig. 5), or a larger body which contained a mitochondrion and micronemes (Fig. 6).

The cross-section of a premature microgamont (Fig. 7) contained more than nine nuclei aligned along the periphery; several formations of centrioles could be detected along the microgamont's boundary. The cyto-

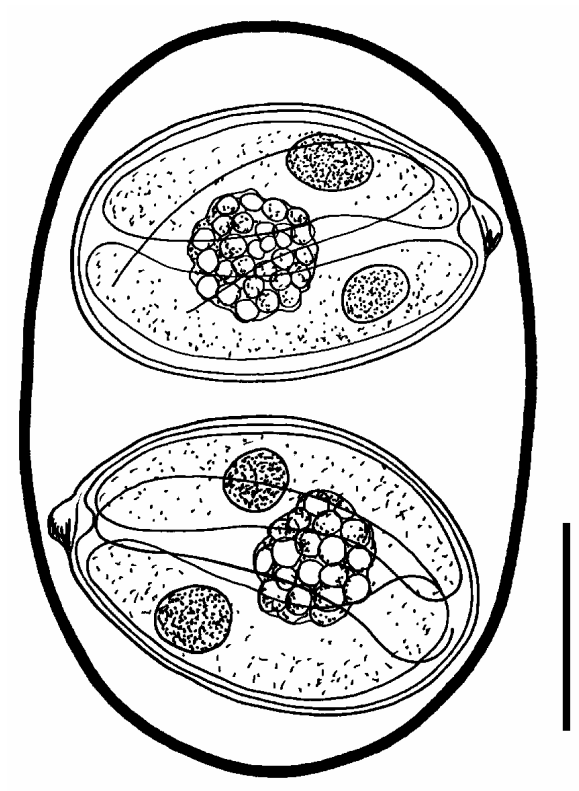

Fig. 1. Oocyst of Isospora carliae sp. n. Scale bar $=5 \mu \mathrm{m}$.

plasm also contained many variable-sized lipid vacuoles, as well as elongate vacuoles - seemingly extracted amylopectin granules. The mature microgamont contained small number of microgametes (Fig. 8). The microgamete cross-section disclosed longitudinal and cross-sections of nuclei, associated mitochondria and a couple of flagella. TEM-examined material also contained microgamonts with numerous microgametes, but is not shown here due to poor image quality.

Macrogamonts (Fig. 9) were bound by a two-layered wall; their dense cytoplasm contained an extensive network of endoplasmic reticulum, lipid vacuoles, amylopectin granules, a few canaliculi, and two types of wall-forming bodies (WFB type-1 and WFB type-2). Mitochondria were aligned along the cell wall and the adnuclear bodies on the periphery of the nucleus. In the zygote or young oocyst (Fig. 10), the wall membrane had doubled and separated, forming two loose envelopes above the plasmalemma (Figs. 10, 11). The cytoplasm was filled with amylopectin bodies, along with large aggregates of canaliculi. A few lipid vacuoles appeared to persist. Young oocysts contained many intact WFB1, while the WFB2 were beginning to fractionate or disintegrate.

T y p e h o s t: The blue-throated rainbow skink, Carlia rhomboidalis (Peters), (Sauria, Scincidae).

Host identified by: Dr. Steve Donellan, South Australia Museum, Adelaide, South Australia.

T y p e 1 o c a 1 i t y: Daintree Forest, North Queensland, Australia $\left(17.5^{\circ} \mathrm{S}, 145.5^{\circ} \mathrm{E}\right)$.

V o u cher m at e ria 1: Semithin sections prepared from Agar $100 \AA$-embedded tissue, deposited in the Israel National Collection of Natural History (parasitological collection, c/o I. Paperna). Oocysts preserved in faecal material stored in $10 \%$ BNF became disintegrated.

E t y m o lo g y : The species is named after the host's generic name. 


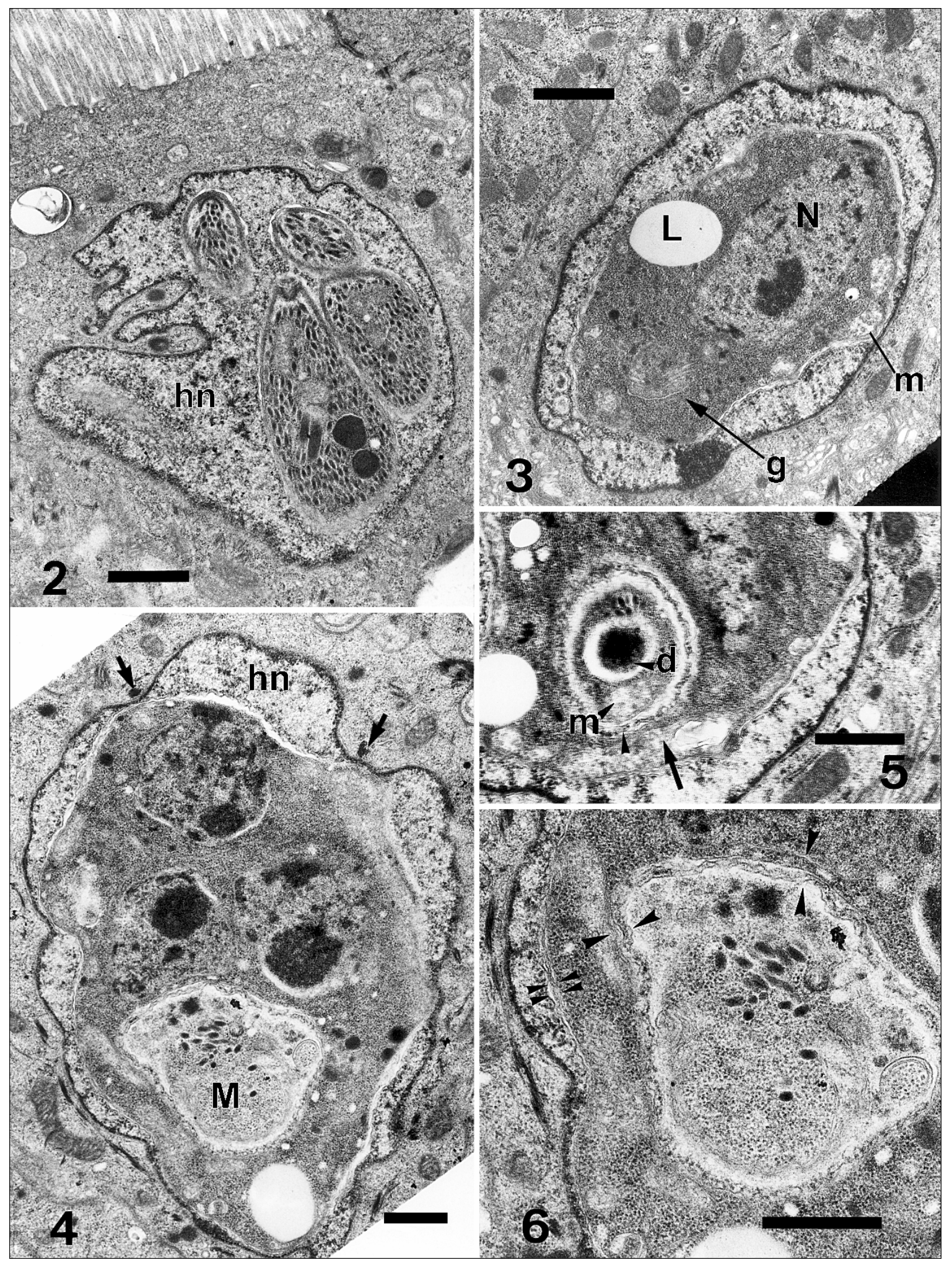

Figs. 2-6. Fine-structural images of merogonic stages of Isospora carliae sp. n. Fig. 2. Host nucleus (hn) invaded by four merozoites. Fig. 3. Young intranuclear meront with a single nucleus $(\mathrm{N})$, showing Golgi elements (g), mitochondria $(\mathrm{m})$ and a lipid vacuole (L) in the cytoplasm. Fig. 4. Dividing meront. The host nucleus (hn) forms junction zones (arrows). The meront contains a membrane-bound inclusion with micronemes (M). Fig. 5. A view of the membrane-bound enclave (arrow) in a different meront. The enclosed membrane-bound (arrowhead) body shows micronemes, a mitochondrion $(\mathrm{m})$ and an electron-dense core (nucleus?) (d). Fig. 6. Enlarged view of the included body shown in Fig. 4, with a closer look at the enclave wall (large arrowheads) as compared to the meront boundary and its parasitophorous wall membranes (small arrowheads). Scale bars $=1 \mu \mathrm{m}$. 


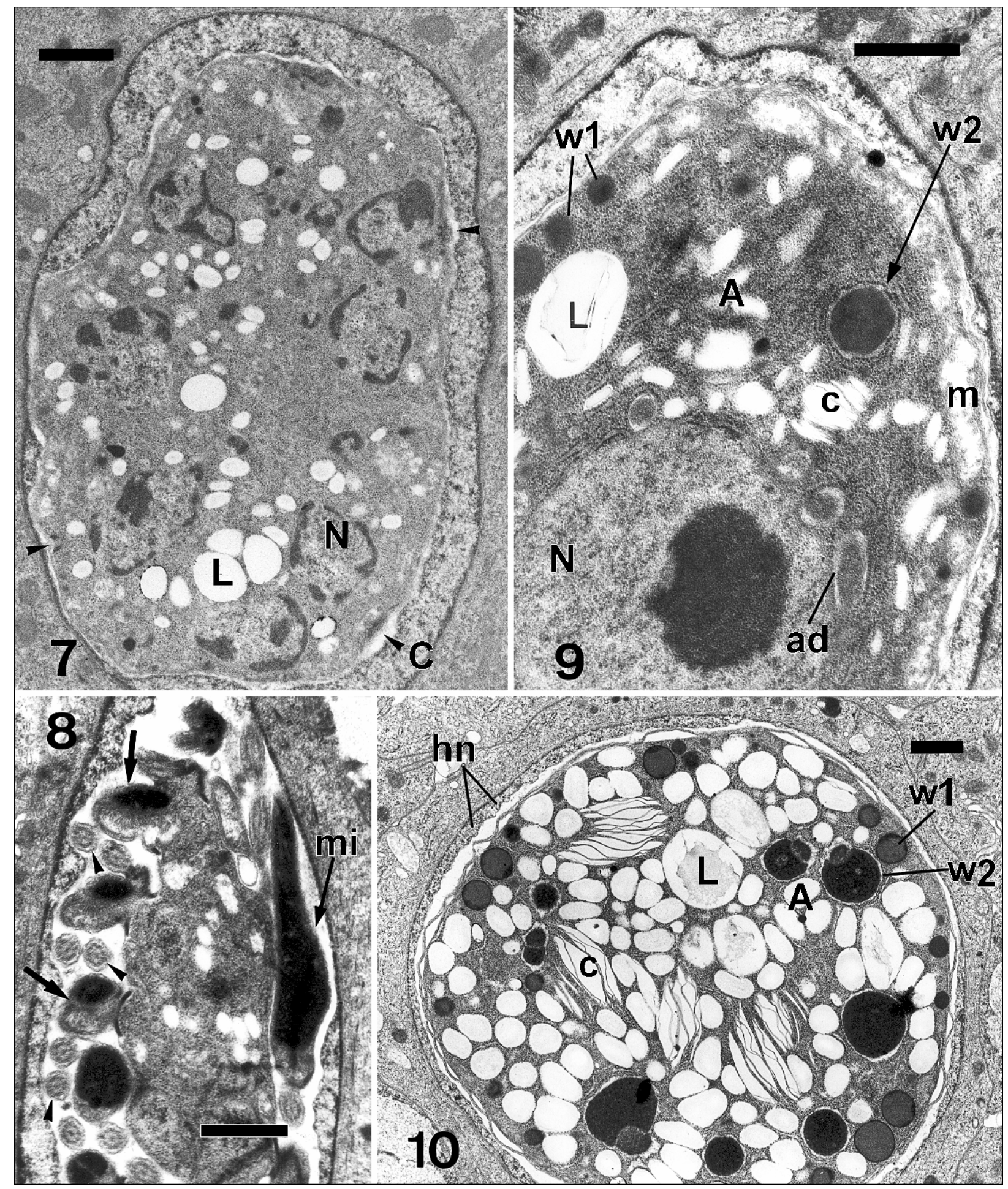

Figs. 7-10. Gamogony stages of Isospora carliae sp. n. Fig. 7. Premature microgamonts with peripherally located nuclei (N) and adjoining sites of forming centrioles (C and arrowheads). Cytoplasm contains numerous lipid vacuoles (L). Fig. 8. Fully differentiated microgamont showing emerging microgametes (arrows) (mi, in longitudinal section) and their flagella (arrowheads). Fig. 9. Macrogamont with a nucleus $(\mathrm{N})$, adnuclear bodies $(\mathrm{ad})$, amylopectin bodies (A), a lipid vacuole (L), mitochondria (m), wall-forming bodies (WFB) type-1 (w1) and type-2 (w2) and canaliculi (c). Fig. 10. Zygote or young oocyst within a residual host nucleus (hn), filled with amylopectin granules (A) and canaliculi (c), with a few lipid vacuoles and WFB type-1 (w1) and type-2 (w2). Scale bars $=1 \mu \mathrm{m}$. 


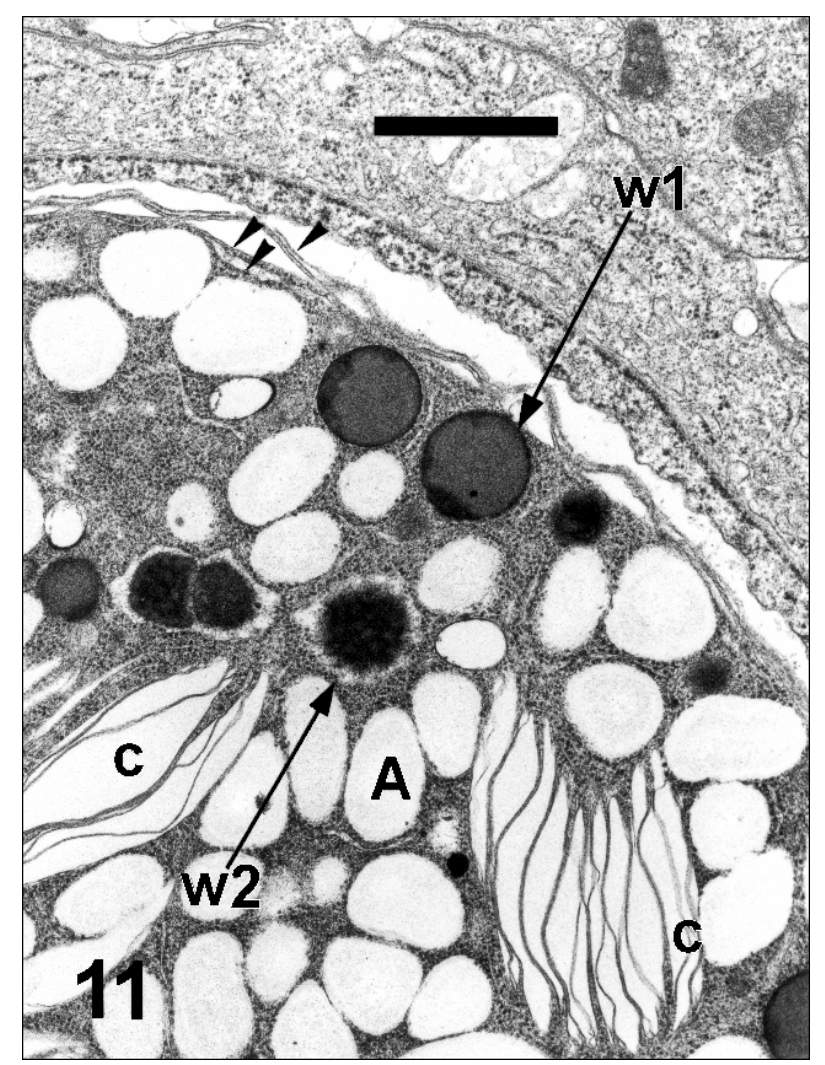

Fig. 11. Zygote or young oocyst of Isospora carliae sp. $\mathrm{n}$. Enlarged sector of Fig. 10 showing the duplication of the wall membranes (arrowheads) (other abbreviations as in Fig. 10). Scale bar $=1 \mu \mathrm{m}$.

\section{DISCUSSION}

Although intranuclear development is a relevant diagnostic characteristic for differentiating Isospora species of reptiles, many of the species descriptions lack information on endogenous development (see Duszynski and Wilber 1997). Of the three species of Isospora described from Australian skinks (a fourth species was reported from skinks by Finkelman and Paperna 1994 by mistake: its host, Delma nasuta, belongs to the family Pygopodidae), only I. cryptoblephari Finkelman et Paperna, 1994 from Cryptoblepharus virgatus from central Queensland exhibited intranuclear development. In the others (I. egerniae Cannon, 1967 and I. ablephari Cannon, 1967), endogenous development occurs in the cytoplasm. Oocysts, as well as all endogenous stages of I. cryptoblephari, are considerably larger than the same stages described here. The ellipsoid oocysts with sporocysts in a "lateral position" distinguish I. carliae from most of the lacertilian Isospora species, in which the oocyst is approximately spherical; this includes all other known species from Australian lizards (Cannon 1967, Finkelman and Paperna 2002) and other known species from skinks elsewhere (Matuschka 1989, El Toukhy et al. 1996), with the exception of I. manchacensis Atkin- son et Ayala, 1987 from the American skink Scincella lateralis, which undergoes intranuclear development and whose oocysts are larger than those described herein.

Paperna and Finkelman, (1998) previously noted that the endogenous stages of the species of Isospora found in Carlia rhomboidalis, here described, are clearly distinguishable from other intranuclear Isospora spp. from other lizards in their smaller sizes, their configuration, small number of progeny of merozoites (about 12; see Paperna and Finkelman 1998, fig. 22, p. 289) and generations of microgamonts with either few or numerous microgametes. The immature merogonic and gamogonic stages are oblong and enclosed within a thicker envelope of host nucleoplasm. The host cell nucleoplasm is retained to a later stage of development and consists of coarser granulation when compared with images of other intranuclear Isospora, even from the same processing batch (I. cannoni from the agamid Diplopora australis, and I. nucleoheteronotis Finkelman et Paperna, 2002 from Heteronotia binoei [= Isospora sp. in Paperna and Finkelman 1998]). Such coarse granulation of the host nucleoplasm is seen in Isospora-infected nuclei from the skink Chalcides ocellatus (El Toukhy et al. 1996).

In I. carliae, a junction zone can be traced between the young meront's parasitophorous vacuole wall and the nucleolemma. This junction, apparently an hemidesomosal structure, occurs in intranuclear-developing coccidia of fish, Isospora of lizards (Paperna and Finkelman 1998, Paperna and Vilenkin 1999) and Tyzzeria of geese (Shibalova and Morozova 1979). The hemidesmosomal structure might facilitate metabolic exchange between the parasite and the host cytoplasm.

The presence of more than one parasite within an infected nucleus, each within its own parasitophorous vacuole, suggests their concomitant entry into the nucleus (also seen in I. cannoni, see Paperna and Finkelman 1998), rather than their being the product of merogonic division, as seen in other instances (I. nucleoheteronotis [= Isospora $\mathrm{sp}$. in Paperna and Finkelman 1998]). The presence of no more than a single mature merogonic or gamogonic stage in intranuclear species of Isospora suggests that multiple infections do not persist to maturity (Paperna and Lainson 2000).

Another finding not mentioned previously is the membrane-enclosed body containing micronemes seen in some premature meronts. The presence of micronemes suggests either hyperinfection by a merozoite, or a deep invagination in the meront's own cytoplasm.

Acknowledgements. I wish to thank Prof. Bruce Copemann of the Graduate School for Tropical Veterinary Science at James Cook University of North Queensland, Australia, for his hospitality and support. Permission to collect was granted from Queensland National Parks and Wildlife Service. 
ATKINSON C.T., AYALA, S.C. 1987: Isospora manchacensis n. sp., an intranuclear coccidian from the Louisiana ground skink, Scincella lateralis (Say, 1823) (Lacertilia Scincidae). J. Protozool. 73: 817-823.

CANNON L.R.G. 1967: New coccidian from Australian lizards. I. Isospora. Parasitology 57: 227-235.

DUSZYNSKI D.W., WILBER P.G. 1997: A guideline for the preparation of species descriptions in the Eimeriidae. J. Parasitol. 83: 333-336.

EL TOUKHY A.A.G., ABDEL-AZIZ A., ABDEL-GAWAD M.A. 1996: Light and electron microscopic studies of Isospora chalcidis (Amoudi, 1989) infecting Chalcides ocellatus in Egypt. J. Egypt. Soc. Parasitol. 26: 79-91.

FINKELMAN S., PAPERNA I. 1994: The endogenous development of two new species of Isospora from skinks. Syst. Parasitol. 27: 227-235.

FINKELMAN S., PAPERNA I. 2002: The endogenous development of four new species of Isospora Schneider, 1881

Received 20 March 2006
(Apicomplexa: Eimeriidae) from Australian geckoes. Syst. Parasitol. 51: 59-71.

MATUSCHKA F.R. 1989: Isospora viridanae n. sp., an intranuclear coccidian parasite from the Canarian skink, Chalcides viridanus (Larcertilia: Scincidae). J. Parasitol. 36: 274-279.

PAPERNA I., FINKELMAN S. 1998: The fine structure of reptilian Isospora species with intranuclear development. Parassitologia 40: 283-296.

PAPERNA I., LAINSON R. 2000: The fine structure of the endogenous stages of Isospora hemidactyli Carini, 1936 in the gecko Hemidactylus mabouia from North Brazil. Mem. Inst. Oswaldo Cruz 95: 43-47.

PAPERNA I., VILENKIN M. 1999: Parasitophorous vacuole of intranuclear coccidian maintains membranous junction with the host cell cytoplasm. Folia Parasitol. 46: 155-156.

SHIBALOVA T.A., MOROZOVA T.I. 1979: Intranuclear development of macrogametes in the coccidium Tyzzeria parvula. Tsitologia (Leningrad) 21: 969-972.

Accepted 14 June 2006 